\title{
Robotic-assisted versus laparoscopic colorectal surgery: a meta-analysis of four randomized controlled trials
}

\author{
Guixiang Liao', Zhihong Zhao ${ }^{2}$, Shuhui Lin ${ }^{1}$, Rong Li ${ }^{1}$, Yawei Yuan ${ }^{1 *}$, Shasha Du', Jiarong Chen ${ }^{1}$ and Haijun Deng ${ }^{3}$
}

\begin{abstract}
Background: Robotic-assisted laparoscopy is popularly performed for colorectal disease. The objective of this meta-analysis was to compare the safety and efficacy of robotic-assisted colorectal surgery (RCS) and laparoscopic colorectal surgery (LCS) for colorectal disease based on randomized controlled trial studies.

Methods: Literature searches of electronic databases (Pubmed, Web of Science, and Cochrane Library) were performed to identify randomized controlled trial studies that compared the clinical or oncologic outcomes of RCS and LCS. This meta-analysis was performed using the Review Manager (RevMan) software (version 5.2) that is provided by the Cochrane Collaboration. The data used were mean differences and odds ratios for continuous and dichotomous variables, respectively. Fixed-effects or random-effects models were adopted according to heterogeneity.

Results: Four randomized controlled trial studies were identified for this meta-analysis. In total, 110 patients underwent RCS, and 116 patients underwent LCS. The results revealed that estimated blood losses (EBLs), conversion rates and times to the recovery of bowel function were significantly reduced following RCS compared with LCS. There were no significant differences in complication rates, lengths of hospital stays, proximal margins, distal margins or harvested lymph nodes between the two techniques.

Conclusions: RCS is a promising technique and is a safe and effective alternative to LCS for colorectal surgery. The advantages of RCS include reduced EBLs, lower conversion rates and shorter times to the recovery of bowel function. Further studies are required to define the financial effects of RCS and the effects of RCS on long-term oncologic outcomes.
\end{abstract}

Keywords: Colorectal, Robotic, Laparoscopic, Meta-analysis, Colorectal cancer

\section{Background}

Minimally invasive surgery for colorectal disease was introduced in 1991 [1]. Such surgery has been widely used worldwide and has become increasingly popular. However, technical barriers, including unstable video camera imaging, loss of dexterity and a steep learning curve, preclude the widespread adoption of minimally invasive surgery techniques for colorectal disease. To overcome these technical drawbacks, robotic surgical systems were introduced. Robotic laparoscopic colorectal

\footnotetext{
*Correspondence: yuanyw12@163.com

'Department of Radiation Oncology, Nanfang Hospital, Southern Medical University, No. 1838, Guangzhou Avenue North, Guangzhou 510515, China Full list of author information is available at the end of the article
}

surgery (RCS) was first reported in 2002 [2]. Since then, many studies of RCS have been widely reported [3-5]. RCS has some advantages over conventional laparoscopic surgery (LCS). These advantages include a three-dimensional image, convenient movements of the robotic arm, no tremor, motion scaling, a short learning curve, dexterity and ambidextrous capability $[4,6,7]$. Some studies have already proven that robotic laparoscopic colorectal surgery is associated with certain benefits compared to conventional laparoscopic colorectal surgery [8-10]. Additionally, the learning curve for robotic surgery is short [11]. However, the relative merits of RCS versus LCS are controversial.

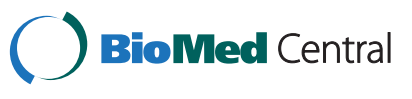

(c) 2014 Liao et al.; licensee BioMed Central Ltd. This is an Open Access article distributed under the terms of the Creative Commons Attribution License (http://creativecommons.org/licenses/by/2.0), which permits unrestricted use, distribution, and reproduction in any medium, provided the original work is properly credited. The Creative Commons Public Domain Dedication waiver (http://creativecommons.org/publicdomain/zero/1.0/) applies to the data made available in this article, unless otherwise stated. 


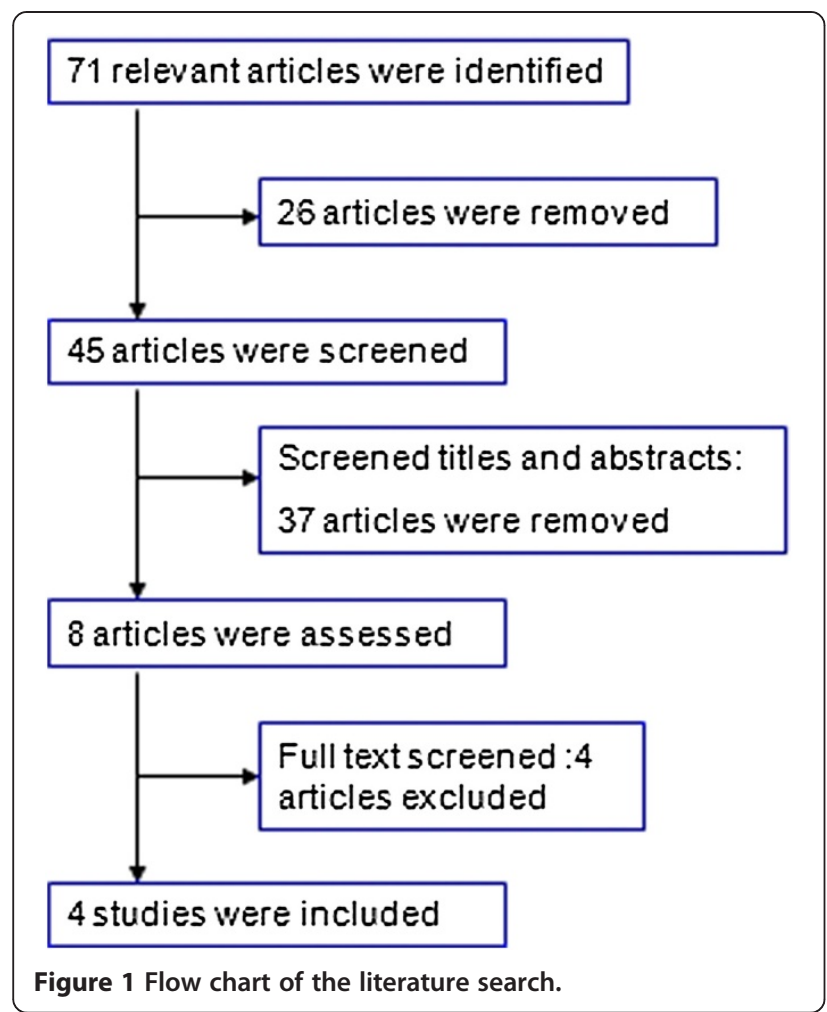

The aim of this study was to compare the efficacy and safety of RCS and LCS via a meta-analysis of the available randomized controlled trials (RCTs).

\section{Methods}

Research method

Two authors (XGL, HZZ) participated in this search to independently identify all published RCT studies comparing RCS and LCS. The Pubmed, Web of Science and Cochrane Library databases were searched. The following medical subject heading terms and key words were used: robotic; telerobotic; colorectal; colon; rectal; random. None of the searches had any language limitations, and the most recent search time was 1 March 2013.

\section{Inclusion criteria}

For inclusion in this meta-analysis, the studies were required to meet the following criteria: (1) RCTs; (2) studies comparing the efficacy and safety of RCS and LCS for the treatment of colorectal disease; (3) studies that effectively reported both primary results and data adequate for meta-analysis.

\section{Data extraction}

The effective data from all eligible RCTs were extracted by two reviewers (XGL, HZZ). The extracted data included the following: first author; year and country of publication; surgical technique; operation type; basic patient characteristics, including age, body mass index (BMI), number of patients, diagnosis, tumor stage, intraoperative data, postoperative data; and pathological details including operation time, estimated blood loss, open conversion rate, time to recovery of bowel function, length of hospital stay, complication rate, proximal margin, distal margin, harvested lymph nodes and cost. All available data were extracted from the relevant texts, tables and figures. In cases of disagreement about whether a study was suitable for inclusion in the meta-analysis, an additional reviewer (WYY) assessed the article and made the final decision.

\section{Risk of bias}

Two reviewers (XGL, HZZ) independently evaluated the bias of each study using the Cochrane tools [12]. The assessment item included sequence generation, allocation of sequence concealment, blinding of participants and personnel, blinding of outcomes and assessments, incomplete outcome data, selective outcome reporting and

Table 1 General characteristics of the studies and participants

\begin{tabular}{|c|c|c|c|c|c|c|c|c|c|c|c|}
\hline Author & Year & Country & Group & $\mathrm{N}$ & $\begin{array}{l}\text { Sex } \\
\text { M/F }\end{array}$ & BMI & $\begin{array}{l}\text { ASA grade } \\
\text { I/II/III/IV }\end{array}$ & Age, years & Disease & $\begin{array}{l}\text { Tumor stage } \\
\text { I/ II/III/IV }\end{array}$ & Operation type \\
\hline \multirow[t]{2}{*}{ Baik SH } & \multirow[t]{2}{*}{2008} & \multirow[t]{2}{*}{ Korea } & $\mathrm{RCS}$ & 18 & $14 / 4$ & $22.8(1.8)$ & $12 / 6 / 0 / 0$ & $57.3(6.3)$ & \multirow[t]{2}{*}{ Rectal cancer } & $5 / 4 / 9 / 0$ & \multirow{2}{*}{$\begin{array}{l}\text { Tumor-specific } \\
\text { mesorectal excision }\end{array}$} \\
\hline & & & LCS & 18 & $14 / 4$ & $24.0(2.5)$ & $9 / 6 / 1 / 1$ & $62.0(9.0)$ & & $5 / 4 / 9 / 0$ & \\
\hline \multirow[t]{2}{*}{ Jimenez RR } & \multirow[t]{2}{*}{2011} & \multirow[t]{2}{*}{ Spain } & $\mathrm{RCS}$ & 28 & $12 / 16$ & $28.59(2.5)$ & $14 / 14^{a}$ & $68.0(9.1)$ & \multirow{2}{*}{$\begin{array}{l}\text { Sigmoid colon cancer } \\
\text { and rectal cancer }\end{array}$} & $15 / 13^{b}$ & \multirow{2}{*}{$\begin{array}{l}\text { Total or subtotal } \\
\text { mesorectal excisior }\end{array}$} \\
\hline & & & LCS & 28 & $17 / 11$ & $26.75(5.6)$ & $20 / 8^{a}$ & $61.5(15.0)$ & & $21 / 7^{b}$ & \\
\hline \multirow[t]{2}{*}{ Park JS } & \multirow[t]{2}{*}{2012} & \multirow[t]{2}{*}{ Korea } & $\mathrm{RCS}$ & 35 & $14 / 21$ & $24.4(2.5)$ & $15 / 16 / 4 / 0$ & $62.8(10.5)$ & \multirow{2}{*}{$\begin{array}{l}\text { Right-sided colon } \\
\text { carcinoma }\end{array}$} & $10 / 16 / 9 / 0$ & \multirow[t]{2}{*}{ Right colectomies } \\
\hline & & & LCS & 35 & $16 / 19$ & $23.8(2.7)$ & $21 / 12 / 2 / 0$ & $66.5(11.4)$ & & $9 / 16 / 10 / 0$ & \\
\hline \multirow[t]{2}{*}{ Patriti A } & \multirow[t]{2}{*}{2009} & \multirow[t]{2}{*}{ Italy } & $\mathrm{RCS}$ & 29 & $11 / 18$ & $24(6.2)$ & $2 / 13 / 14 / 0$ & $68(10)$ & \multirow[t]{2}{*}{ Rectal cancer } & $11 / 9 / 7 / 2$ & \multirow{2}{*}{$\begin{array}{l}\text { Total mesorectal } \\
\text { excision }\end{array}$} \\
\hline & & & LCS & 37 & $12 / 25$ & $25.4(6.44)$ & $2 / 14 / 21 / 0$ & $69(10)$ & & $17 / 8 / 10 / 2$ & \\
\hline
\end{tabular}


Table 2 Data extracted from the included studies

\begin{tabular}{|c|c|c|c|c|c|c|c|c|c|c|c|c|}
\hline Study & Group & $\mathbf{N}$ & $\begin{array}{l}\text { Operation time, } \\
\text { minutes }\end{array}$ & $\mathrm{EBL}, \mathrm{ml}$ & $\begin{array}{l}\text { Open conversion } \\
\text { events, } n\end{array}$ & $\begin{array}{l}\text { Time to recovery of } \\
\text { bowel function, } d\end{array}$ & $\begin{array}{l}\text { Complication } \\
\text { events, } n\end{array}$ & LOS, d & $\begin{array}{l}\text { Proximal } \\
\text { margin, } \mathrm{cm}\end{array}$ & Distal margin, $\mathrm{cm}$ & $\begin{array}{l}\text { Harvested lymph } \\
\text { nodes, } \mathrm{n}\end{array}$ & $\begin{array}{l}\text { Total hospital } \\
\text { cost, } \$\end{array}$ \\
\hline \multirow[t]{2}{*}{ Baik SH } & RCS & 18 & $217.1(51.6)$ & N/A & 0 & $1.8(0.4)$ & 4 & $6.9(1.3)$ & $10.9(1.7)$ & $4(1.1)$ & $20.1(9.1)$ & N/A \\
\hline & LCS & 18 & $204.3(56.9)$ & & 2 & $2.4(1.3)$ & 1 & $8.7(1.7)$ & $10.3(3.6)$ & $3.7(1.1)$ & $17.4(10.6)$ & \\
\hline \multirow[t]{2}{*}{ Jimenez RR } & RCS & 28 & $159.4(43.5)$ & N/A & 2 & N/A & 4 & N/A & N/A & $4.8(1.6)$ & $17.6(9.2)$ & N/A \\
\hline & LCS & 28 & $135.1(29.2)$ & & 2 & & 4 & & & $3.8(0.7)$ & $14.9(8.7)$ & \\
\hline \multirow[t]{2}{*}{ Park JS } & RCS & 35 & $195(41)$ & $35.8(26.3)$ & 0 & $2.6(1.4)$ & 6 & $7.9(4.1)$ & $18.6(7.3)$ & $18(9)$ & $29.9(14.7)$ & $12,235(1,907.9)$ \\
\hline & LCS & 35 & $130(43)$ & $56.8(31.3)$ & 0 & $2.9(2.2)$ & 7 & $8.3(4.2)$ & $18.3(9.9)$ & $14.5(8)$ & $30.8(13.3)$ & $10,320.7(1,607.7)$ \\
\hline \multirow[t]{2}{*}{ Patriti A } & RCS & 29 & $202(12)$ & $137.4(156)$ & 0 & N/A & 9 & 11.9 (7. 5) & N/A & $2.1(0.9)$ & $10.3(4)$ & N/A \\
\hline & LCS & 37 & $208(7)$ & 127 (169) & 7 & & 7 & $9.6(6.9)$ & & $4.5(7.2)$ & $11.2(5)$ & \\
\hline
\end{tabular}

Values are expressed as mean (SD) or number. EBL, estimated blood loss; LOS, length of hospital stay; N/A, not applicable; RCS, robotic-assisted colorectal surgery; LCS, laparoscopic colorectal surgery. 


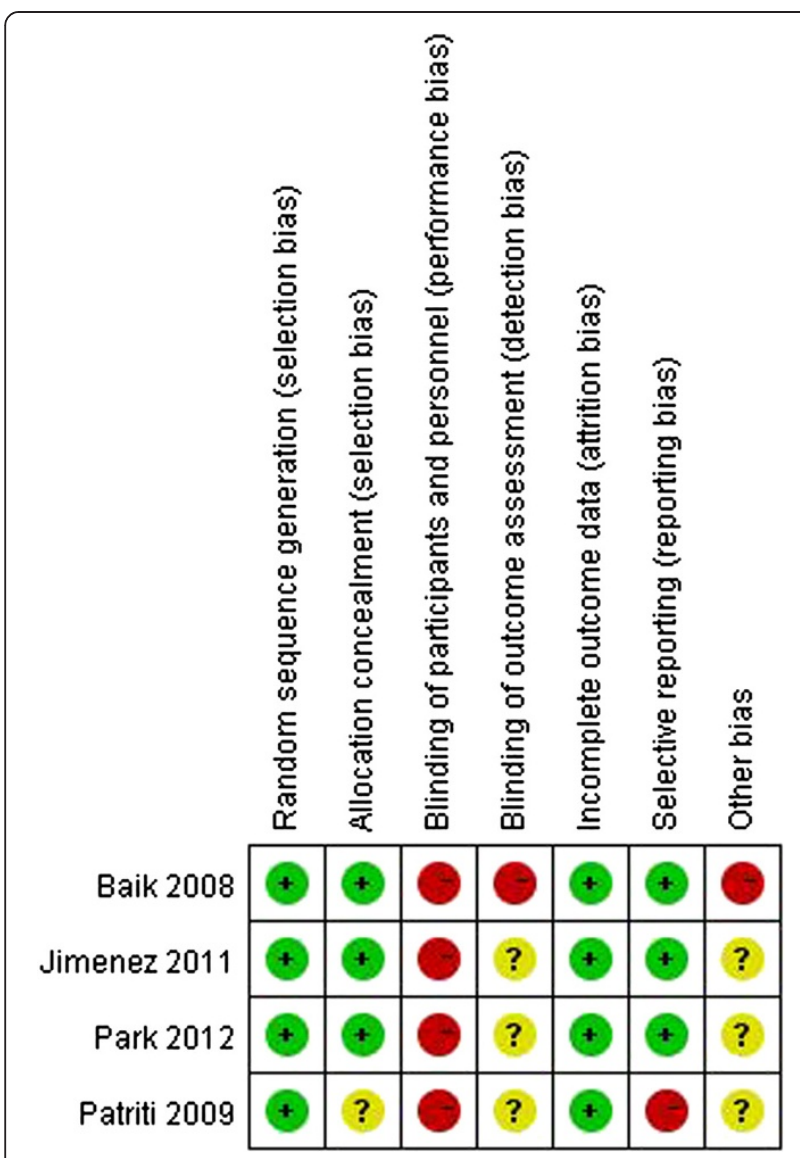

Figure 2 Risk of bias graph. (+), low risk of bias; (-), high risk of bias; (?), unclear risk of bias.

other biases. When disagreement existed, the third reviewer (WYY) discussed the issues with the group and made a final conclusion.

\section{Statistical analyses}

This meta-analysis was performed using the Review Manager (RevMan) software, version 5.2, provided by the Cochrane Collaboration. Dichotomous variables were analyzed using estimations of the odds ratio (OR) and $95 \% \mathrm{CI}$, and continuous variables were analyzed using weighted mean difference (WMD) and 95\% CI. Fixed-effects models or random-effects models were applied. Heterogeneity was evaluated using $X^{2}$ and $I^{2}$ tests. We considered heterogeneity to be present when the $I^{2}$ statistic was $>50 \%$, and random-effects models were adopted in these cases. However, when the $I^{2}$ statistic was $<50 \%$, we used fixed-effects models. $P<0.05$ was considered significant.

\section{Results}

\section{Literature search results}

A total of 71 potentially relevant articles were identified, and the selection process is illustrated in Figure 1. After examination of the titles, abstracts and full texts, four published RCT studies [13-16] were found to meet all of the inclusion criteria and were entered into this metaanalysis. In total, 110 patients underwent RCS, and 116 patients underwent LCS. One study of right-sided colonic carcinoma [13], two studies of rectal cancer $[14,15]$, and another study that included sigmoid colon cancer and rectal cancer [16] were reported. Information about the general characteristics of the studies and the participants is listed in Table 1, and the extracted data are shown in Table 2.

\section{Risk of bias assessment}

All of the studies were RCTs, but none were double- or even single-blinded. Assessments of the risk of bias are illustrated in Figure 2 and Figure 3.

\section{Meta-analysis \\ Operation times}

All four of the studies reported operation times. Two of the four studies indicated a tendency toward longer operation times for the RCS groups than for the LCS

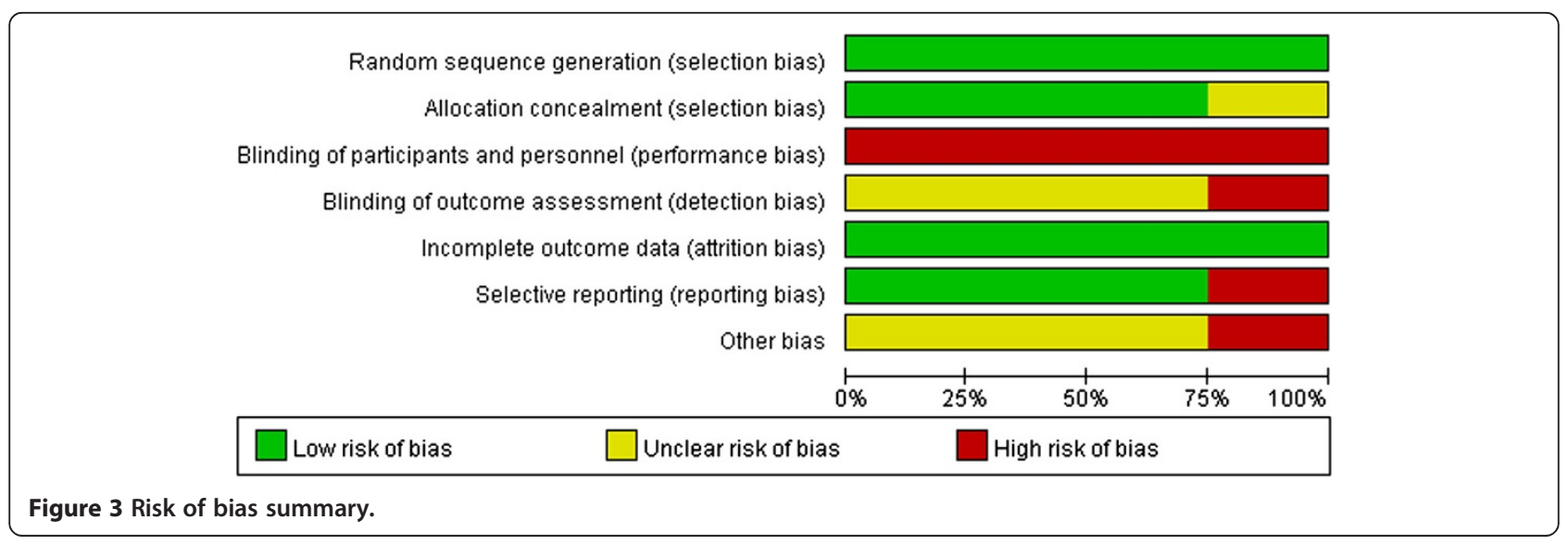




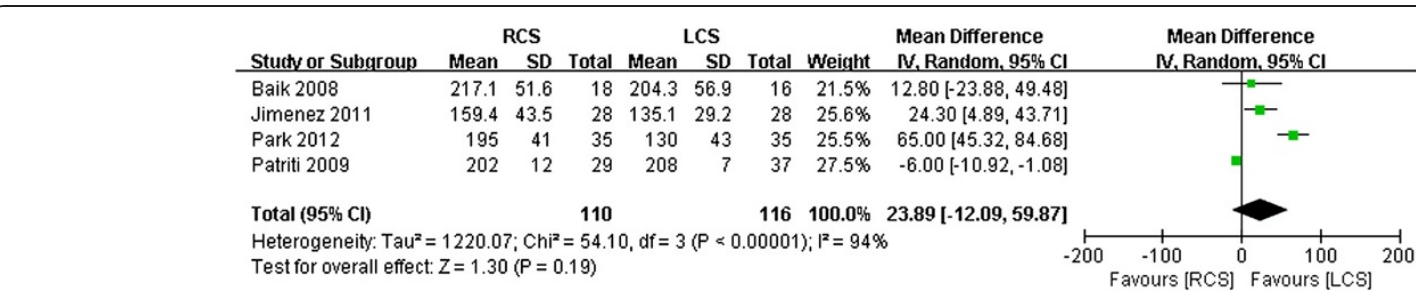

Figure 4 Meta-analysis of the operation times shown as a forest plot between robotic-assisted colorectal surgery (RCS) and laparoscopic colorectal surgery (LCS). The mean differences with 95\% Cls are shown.

groups $[13,16]$. However, no significant differences between the two groups were reported in the other two studies $[14,15]$. The pooled data revealed that the operative times were not significantly different between the two techniques (WMD 23.89, 95\% CI 12.09 to $59.87 ; P=0.19$ ), but the heterogeneity was high $\left(P<0.001, I^{2}=94 \%\right)$ (Figure 4).

\section{Estimated blood losses}

Only two of the studies described intraoperative estimated blood losses (EBLs) [13,14]. Both of these studies reported that the EBLs did not significantly differ between the two approaches. In the studies of Baik et al., the hemoglobin change $(\mathrm{g} / \mathrm{dl})$ indices before and after the surgical inventions were reported. These numbers revealed no significant difference between the two techniques [15]. The pooled data from these studies suggested that the EBL of the RCS group was significantly lower than that of the LCS group (WMD -20.10, 95\% CI -33.44 to $-6.75 ; P=0.003)$. Furthermore, there was no evidence of observed heterogeneity $\left(P=0.59, I^{2}=0 \%\right)$ (Figure 5).

\section{Conversion rate}

Overall, conversions to open operations were required for two patients in the RCS group (1.82\%) and for 11 patients in the LCS group (9.48\%). Analyses of the pooled data revealed that the conversion rate was significantly lower in the RCS group than in the LCS group (WMD $0.25,95 \%$ CI 0.07 to $0.91 ; P=0.04$ ), and there was no obvious heterogeneity among the four studies $\left(P=0.27, I^{2}=24 \%\right)$ (Figure 6).

\section{Time to recovery of bowel function}

The time to first flatus was reported by Park et al. [13], and the number of days to peristalsis was reported by Baik et al. [15]; both of these variables are indicative of the time to the recovery of bowel function. Analyses of the pool data revealed that the RCS group exhibited shorter times to the recovery of bowel function than did the LCS group (WMD $0.54,95 \%$ CI -0.93 to -0.14 ; $P=0.008, I^{2}=0$ ) (Figure 7 ).

\section{Length of hospital stay}

Four of the studies reported the duration of hospital stays. Baik et al. [15] revealed that the mean length of the hospital stay (LOS) for all patients who underwent laparoscopic procedures was longer than that of those who underwent robotic procedures $(P<0.001)$. However, the other three studies reported no differences in hospital stays between the two groups. Analysis of all of the studies revealed no significant difference between the two techniques in terms of LOS (WMD -0.53, 95\% CI -2.14 to $2.08 ; P=0.52$ ). However, the heterogeneity was slightly high $\left(P=0.19, I^{2}=51 \%\right)$ (Figure 8).

\section{Complication rate}

The complication rates were similar across studies, and there was no significant heterogeneity $\left(I^{2}=0\right)$. A meta-analysis of all of the studies in this index revealed that the complication rates of the two group were not obviously different (odds ratio (OR) 1.39, 95\% CI 0.71 to $2.74, P=0.33$ ) (Figure 9 ).

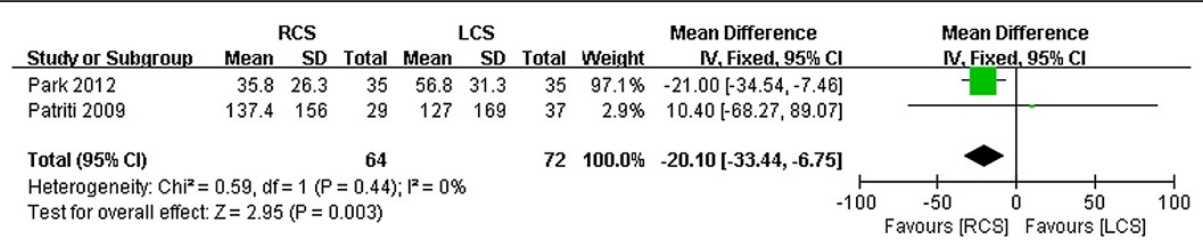

Figure 5 Meta-analysis of the EBLs shown as a forest plot between robotic-assisted colorectal surgery (RCS) and laparoscopic colorectal surgery (LCS). The mean differences with $95 \%$ Cls are shown. 


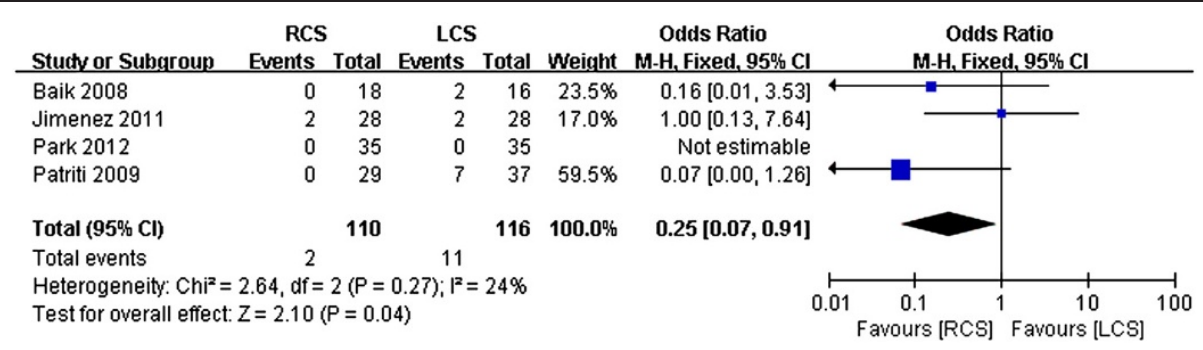

Figure 6 Meta-analysis of the conversion rates shown as a forest plot between robotic-assisted colorectal surgery (RCS) and laparoscopic colorectal surgery (LCS). The odds ratios with $95 \% \mathrm{Cls}$ are shown.

\section{Meta-analysis of the pathological details}

Baik et al. [15] and Park et al. [13] reported proximal margin indices, and there was no difference in this regard (WMD $-0.54,95 \% \mathrm{CI}-1.20$ to $2.29 ; P=0.54$, $I^{2}=0$ ) (Figure 10). All of the studies reported distal margins, and there was no difference between the two groups in this regard (WMD 0.37, 95\% CI - 0.79 to $1.54 ; P=0.53$ ) (Figure 11 ). The studies exhibited significant heterogeneity $\left(I^{2}=71 \%\right)$; one study by Patriti et al. [14] found a high SD for the LCS group $(7.2 \mathrm{~cm})$, and, if this study was excluded, the heterogeneity was relatively low $\left(I^{2}=48 \%\right)$. The harvested lymph nodes were similar across all of the studies $\left(I^{2}=0\right)$, and analysis of the pooled data revealed that the two groups did not differ significantly in this regard (WMD $0.09,95 \%$ CI -1.91 to $1.72 ; P=0.92, I^{2}=0$ ) (Figure 12).

\section{Costs}

As a newly developing surgical technique, it should prove to be safe and cost-effective until it is widely accepted worldwide. Thus, it is essential to evaluate the cost-effectiveness between RCS and LCS. In our meta- analysis, only one study addressed to this issue. Park et al. [13] reported that the overall hospital costs were significantly higher in the RCS group (US \$12235 versus \$10319.7), as showed in Table 2. Based on the limited data, it would be rash to make a conclusion that RCS is not cost-effective.

\section{Publication bias}

Complications were assessed by a funnel plot of the standard errors of the fixed-effect sizes between the RCS and LCS. This analysis revealed no evidence of publication bias or heterogeneity among the studies $(P=0.55$, Figure 13).

\section{Sensitivity analysis}

A sensitivity analysis was performed by excluding the low-quality studies (that is, those with high risk of bias). As shown in Figure 2, we considered the studies of Patriti et al. [14] to have high risks of bias. All variables were included for the sensitivity analysis. If one index was not enough available in a sufficient number of studies (that is, at least two), this index was excluded from further sensitivity analysis. The sensitivity analysis revealed that the operation times of the RCS group were longer than that of the LCS group (WMD 35.97, 95\% CI 3.83 to 68.10, $P=0.03)$. There was no significant difference in the conversion rates of the two techniques (OR 0.51, 95\% CI 0.10 to $2.49 ; P=0.41)$. Analyses of the times to recovery of bowel function, complication rates, proximal margins, distal margins, and the harvested lymph nodes yielded similar results. However, the LOS of the RCS group were significantly shorter than those of the LCS group (WMD $-1.35,95 \% \mathrm{CI}-2.34$ to $-0.37, P=0.007$ ).

\section{Discussion}

Laparoscopic surgery has been applied in many surgical fields and has not only been shown to be effective

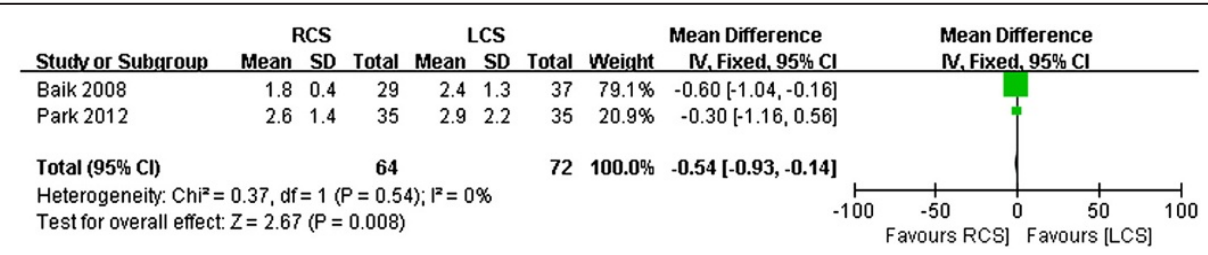

Figure 7 Meta-analysis of the times to the recovery of bowel function shown as a forest plot between robotic-assisted colorectal surgery (RCS) and laparoscopic colorectal surgery (LCS). The mean differences with 95\% Cls are shown. 


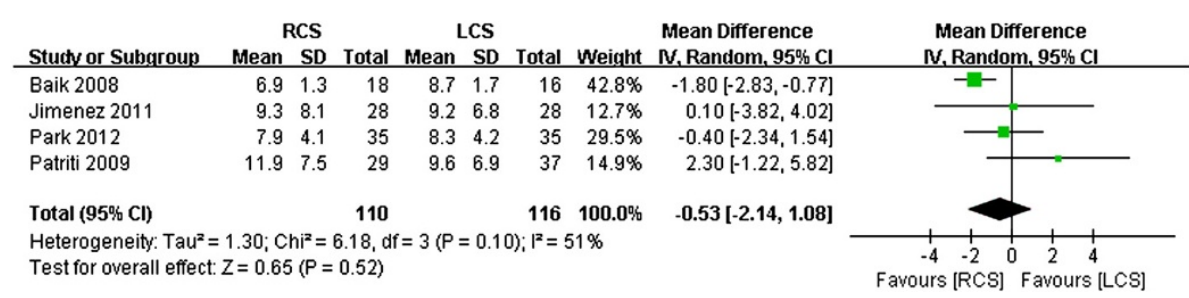

Figure 8 Meta-analysis of length of hospital stays shown as a forest plot between robotic-assisted colorectal surgery (RCS) and laparoscopic colorectal surgery (LCS). The mean differences with 95\% Cls are shown.

and safe but also to have certain benefits over traditional open surgery [17-19]. However, limitations to the performance of laparoscopic surgery remain, including the high conversion rate to open surgery [20]. The advent of robot-assisted surgery has overcome some of the limitations of laparoscopic surgery and has been successfully applied to urology, general surgery, gynecology and other surgical fields [21,22]. Several meta-analyses have suggested that robotic laparoscopic surgery is feasible, produces similar perioperative outcomes and is oncologically safe $[23,24]$. Robotic surgery may result in shorter hospital stays, reduced blood losses and lower conversion rates, but it also may require increased operation time costs [24].

A previous meta-analysis [25] produced a similar conclusion regarding the comparison of robotic laparoscopic surgery to laparoscopic surgery in colorectal disease. However, the studies that were included in this previous meta-analysis were of relatively low quality, and the number of patients was relatively small. Additionally, the benefits of robotic surgery for colorectal disease remain controversial. Thus, we conducted this meta-analysis based on four RCTs to draw a clearer conclusion.

The present study is the first meta-analysis to compare robot-assisted and laparoscopic surgery for colorectal cancer based only on RCTs. The results of this meta-analysis revealed that RCS may provide additional benefits over LCS and that it is safe and effective
$[3,4,26]$. Regarding operation times, RCS exhibited a tendency to take longer than LCS, but this difference was not significant $(P=0.06)$. This result is similar to that of a previous study [27]. In contrast to this view, other studies have reported that RCS requires more time than does LCS $[28,29]$. As the surgeons' robotic experience increases and the techniques are improved, the operation times will be reduced [26]. However, in this regard, obvious heterogeneity existed among the studies; the reasons for this heterogeneity were as follows. First, the surgeries were performed by different surgeons who were not at the same point on the learning curve and had different amounts of experience. Additionally, the RCS group of one study contained a large number of patients who had previously undergone abdominal surgery [14], and it is well-known that previous surgery inevitably increases the difficulty of performing surgery.

EBL ranges between $90 \mathrm{ml}$ and $320 \mathrm{ml}$ for LCS [30] and between $20 \mathrm{ml}$ and $486 \mathrm{ml}$ for RCS according to a recently published review [31]. Our studies showed that the average EBL was $81.83 \mathrm{ml}$ for RCS and $92.88 \mathrm{ml}$ for LCS. Both studies reported no significant difference between the two groups in this regard. However, the pooled data revealed that the EBL was significantly lower for the RCS group than for the LCS group. This finding is consistent with those of previous studies [31]. However, the surgery that was performed by skilled surgical teams exhibited similarly low levels

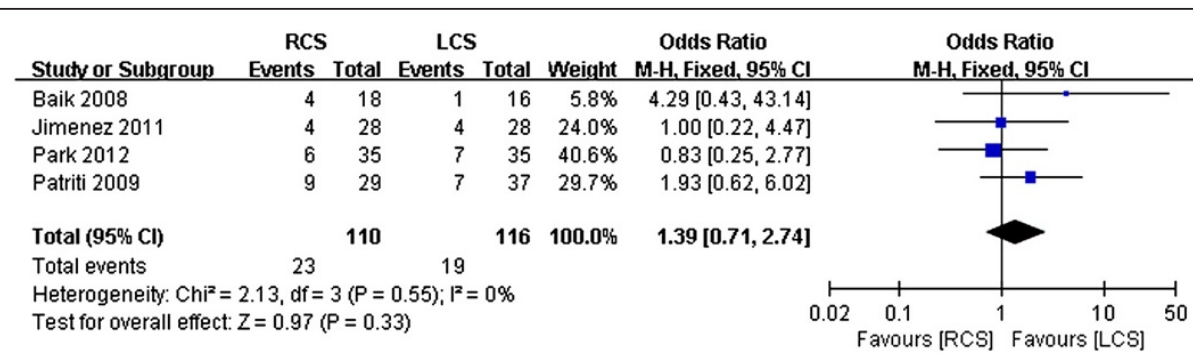

Figure 9 Meta-analysis of the complication rates shown as a forest plot between robotic-assisted colorectal surgery (RCS) and laparoscopic colorectal surgery (LCS). The odds ratios with 95\% Cls are shown. 


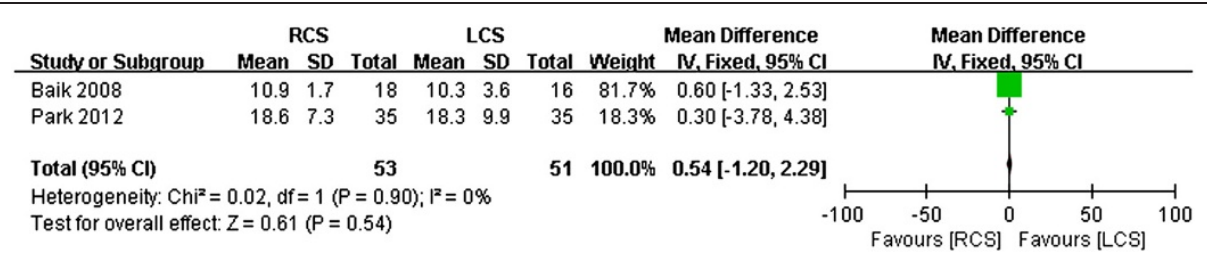

Figure 10 Meta-analysis of the proximal margins shown as a forest plot between robotic-assisted colorectal surgery (RCS) and laparoscopic colorectal surgery (LCS). The mean differences with the $95 \% \mathrm{Cls}$ are shown.

of EBL for the two techniques [4]. Due to lower EBL, it is possible to suggest that RCS may significantly reduce the probability of transfusion and might prevent the recurrence of cancer. A review reported that patients who receive more perioperative transfused blood are at greater risk for cancer recurrence and that this risk is independent of tumor stage at surgery [32]. It is wellknown that the prognosis for colorectal cancer after surgery is highly dependent on the cancer stage at surgery. However, the EBL during colorectal cancer surgery influences long-term survival according to a previous study [33]. Massive blood loss during colorectal cancer surgery is probably related to advanced disease or the recurrence of cancer [34], preoperative therapy (neoadjuvant therapy with either chemoradiation or chemotherapy alone), unclear anatomy [35] and the lack of experience of the surgical team rather than the type of surgical approach. Because only two studies reported EBL in this analysis, further studies are necessary to prove whether RCS results in lower blood loss.

We found that the conversion rate was significantly lower in the RCS group and there was no significant heterogeneity across the four studies. Thus, the reduction in conversion rate may be one of the key benefits of RCS. Conversion rate is a valuable index of surgical quality. Lower conversion rates are associated with fewer postoperative complications [20]. The reduction in conversion rate may be attributable to the fact that robotic surgery can facilitate certain steps in colorectal procedures. These procedures include splenic flexure takedown, dissection of the inferior mesenteric vessels, dissection in the narrow pelvis [36], et cetera.

As observed in the studies, robotic surgery has other advantages. The time to the recovery of bowel function was shorter in the RCS than in the LCS group. This reduction may be another potential benefit of RCS because faster returns to normal diets are associated with faster recoveries. We discovered that the LOS was not significantly different between the two groups; indeed, LOS was similar between the two techniques [26]. The complication rates of the patients who underwent RCS and LCS were similar. This finding also demonstrates that RCS is as safe and feasible as LCS [37]. Regarding the pathological details, there were no differences between the two techniques in terms of proximal margins, distal margins or harvested lymph nodes.

Although we conducted this meta-analysis based on four RCTs, this meta-analysis still has the following limitations. First, the included studies were not doubleblind studies or even single-blind studies; the studies may have been biased; therefore, the interpretation of their results may also have been biased. Second, although we focused on colorectal surgery, different types of diseases (for example, rectal cancer, sigmoid colon cancer, right colon cancer) that involved different types of surgery were included, which may have caused heterogeneity. Third, the surgery in the included cases were not performed by the same surgeon, and the experience and technique of the surgeons may

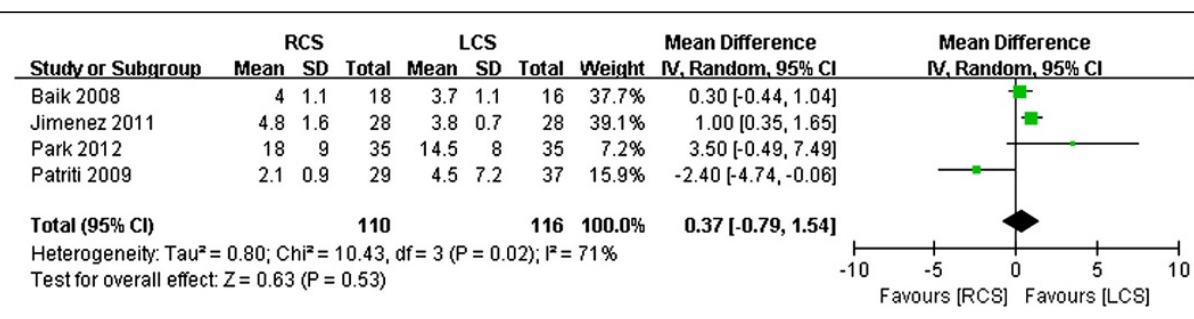

Figure 11 Meta-analysis of the distal margins shown as a forest plot between robotic-assisted colorectal surgery (RCS) and laparoscopic colorectal surgery (LCS). The mean differences with the $95 \% \mathrm{Cls}$ are shown. 


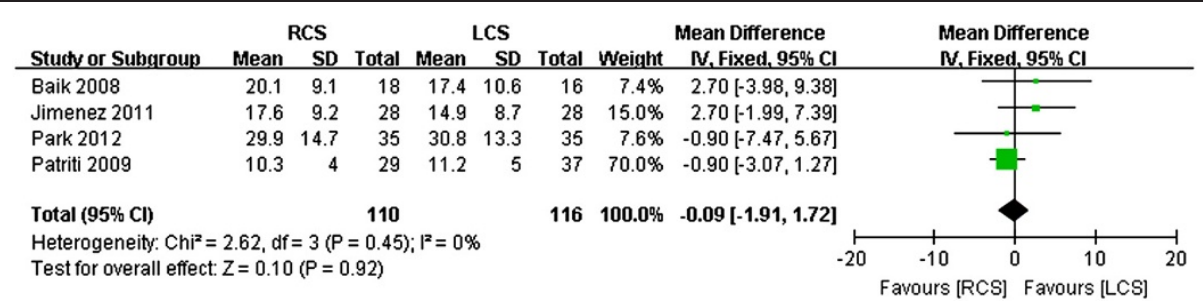

Figure 12 Meta-analysis of the harvested lymph nodes shown as a forest plot between robotic-assisted colorectal surgery (RCS) and laparoscopic colorectal surgery (LCS). The mean differences with the $95 \% \mathrm{Cls}$ are shown.

have affected some of the indices, such as the operation time, EBL and conversion rate. However, in this meta-analysis, no significant heterogeneities among the four studies were found for conversion rate or EBL; only operation time exhibited significant heterogeneity. Fourth, long-term follow-up evaluations assessing oncologic outcomes were not available from the selected studies, and only Patriti et al. [14] reported medium-term outcomes. Fifth, the available included RCT studies were limited, and the numbers of operations were small; however, other RCT studies comparing RCS and LCS, such as the ACOSOG Z6051 and ROLARR studies, are ongoing [38]. Finally, only one study compared costs between RCS and LCS [13]. This study revealed that the overall hospital costs were significantly higher for RCS than for LCS. Robotics is a new trend in colorectal surgery, and more attention has been paid to the high cost that is associated with this new technique. A variety of studies have reported that the cost of robotic colorectal surgery is higher than that of laparoscopic surgery $[3,39,40]$. The high capital and running costs of robotic systems have precluded their widespread use in many countries [41]. Furthermore, one study reported that RCS does not provide sufficient advantages over LCS but significantly increases the total cost [38]. Future studies should assess the cost-effectiveness of RCS based on long-term oncologic outcomes and functional results.

\section{Conclusions}

This meta-analysis suggests that RCS is safe and effective and has some advantages over LCS. These benefits include a reduced conversion rate, reduced blood loss and reduced time to recovery of bowel function. Regarding the other variables, such as operation time, complication rate and LOS, there were no significant differences between the two groups. However, RCS was associated with a significant increase in total costs relative to LCS. Further well-designed, prospective controlled randomized trials should be conducted to assess the financial benefits and the long-term oncologic outcomes of RCS.

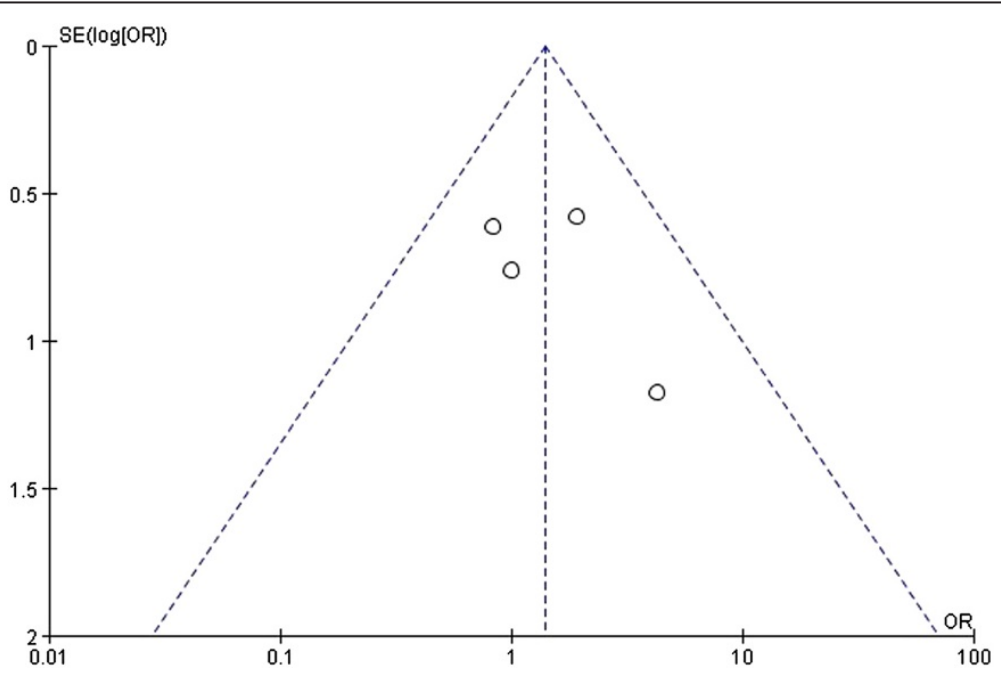

Figure 13 Funnel plot used to assess a fixed-effects model of the results of all of the selected studies regarding the complications of robotic-assisted colorectal surgery (RCS) and laparoscopic colorectal surgery (LCS). SE, standard error. 


\section{Abbreviations}

BMl: body mass index; EBL: estimated blood loss; LCS: laparoscopic colorectal surgery; OR: odds ratio; RCS: robotic-assisted colorectal surgery;

RCT: randomized controlled trial; WMD: weighted mean difference.

\section{Competing interests}

The authors declare that they have no competing interests.

\section{Authors' contributions}

GXL, ZHZ, YWY and SHL conceived the study and participated in its design, the literature research, data extraction, manuscript drafting. RL, JRC, SSD and HJD participated in manuscript drafting, and editing. All authors read and approved the final manuscript.

\section{Acknowledgements}

This study was supported by grants from Guangdong Education University-Industry Cooperation Projects (2011B090400019) and a Natural Science Foundation of China grant (81272508).

\section{Author details}

'Department of Radiation Oncology, Nanfang Hospital, Southern Medical University, No. 1838, Guangzhou Avenue North, Guangzhou 510515, China. ${ }^{2}$ Institute of Nephrology and Urology, Third Affiliated Hospital of Southern Medical University, Guangzhou 510630, China. ${ }^{3}$ Department of General Surgery, Nanfang Hospital, Southern Medical University, No. 1838 Guangzhou Avenue North, Guangzhou 510515, China.

Received: 28 January 2014 Accepted: 9 April 2014

Published: 26 April 2014

\section{References}

1. Jacobs M, Verdeja JC, Goldstein HS: Minimally invasive colon resection (laparoscopic colectomy). Surg Laparosc Endosc 1991, 1:144-150.

2. Weber PA, Merola S, Wasielewski A, Ballantyne GH: Telerobotic-assisted laparoscopic right and sigmoid colectomies for benign disease. Dis Colon Rectum 2002, 45:1689-1694. 1695-1696.

3. Delaney CP, Lynch AC, Senagore AJ, Fazio WW: Comparison of robotically performed and traditional laparoscopic colorectal surgery. Dis Colon Rectum 2003, 46:1633-1639.

4. D'Annibale A, Morpurgo E, Fiscon V, Trevisan P, Sovernigo G, Orsini C, Guidolin D: Robotic and laparoscopic surgery for treatment of colorectal diseases. Dis Colon Rectum 2004, 47:2162-2168.

5. D'Annibale A, Pernazza G, Morpurgo E, Monsellato I, Pende V, Lucandri G, Termini B, Orsini C, Sovernigo G: Robotic right colon resection: evaluation of first 50 consecutive cases for malignant disease. Ann Surg Oncol 2010 17:2856-2862.

6. Park JS, Choi GS, Lim KH, Jang YS, Jun SH: Robotic-assisted versus laparoscopic surgery for low rectal cancer: case-matched analysis of short-term outcomes. Ann Surg Oncol 2010, 17:3195-3202.

7. Baik SH, Kwon HY, Kim JS, Hur H, Sohn SK, Cho CH, Kim H: Robotic versus laparoscopic low anterior resection of rectal cancer: short-term outcome of a prospective comparative study. Ann Surg Oncol 2009, 16:1480-1487.

8. Kim JY, Kim NK, Lee KY, Hur H, Min BS, Kim JH: A comparative study of voiding and sexual function after total mesorectal excision with autonomic nerve preservation for rectal cancer: laparoscopic versus robotic surgery. Ann Surg Oncol 2012, 19:2485-2493.

9. Bianchi PP, Ceriani C, Locatelli A, Spinoglio G, Zampino MG, Sonzogni A, Crosta C, Andreoni B: Robotic versus laparoscopic total mesorectal excision for rectal cancer: a comparative analysis of oncological safety and short-term outcomes. Surg Endosc 2010, 24:2888-2894.

10. Rawlings AL, Woodland JH, Vegunta RK, Crawford DL: Robotic versus laparoscopic colectomy. Surg Endosc 2007, 21:1701-1708.

11. Jimenez-Rodriguez RM, Diaz-Pavon JM, de la Portilla DJF, Prendes-Sillero E, Dussort HC, Padillo J: Learning curve for robotic-assisted laparoscopic rectal cancer surgery. Int J Colorectal Dis 2013, 28:815-821.

12. Higgins JP, Altman DG, Gotzsche PC, Juni P, Moher D, Oxman AD, Savovic J, Schulz KF, Weeks L, Sterne JA: The Cochrane collaboration's tool for assessing risk of bias in randomised trials. BMJ 2011, 343:d5928.

13. Park JS, Choi GS, Park SY, Kim HJ, Ryuk JP: Randomized clinical trial of robot-assisted versus standard laparoscopic right colectomy. $\mathrm{Br} J$ Surg 2012, 99:1219-1226.
14. Patriti A, Ceccarelli G, Bartoli A, Spaziani A, Biancafarina A, Casciola L: Short- and medium-term outcome of robot-assisted and traditional laparoscopic rectal resection. JSLS 2009, 13:176-183.

15. Baik SH, Ko YT, Kang CM, Lee WJ, Kim NK, Sohn SK, Chi HS, Cho CH: Robotic tumor-specific mesorectal excision of rectal cancer: short-term outcome of a pilot randomized trial. Surg Endosc 2008, 22:1601-1608.

16. Jimenez RR, Diaz PJ, de La Portilla DJF, Prendes SE, Hisnard CDJ, Padillo J: Prospective randomised study: robotic-assisted versus conventional laparoscopic surgery in colorectal cancer resection. Cir Esp 2011, 89:432-438

17. Ma Y, Yang Z, Qin H, Wang Y: A meta-analysis of laparoscopy compared with open colorectal resection for colorectal cancer. Med Oncol 2011. 28:925-933.

18. Abraham NS, Young JM, Solomon MJ: Meta-analysis of short-term outcomes after laparoscopic resection for colorectal cancer. $\mathrm{Br}$ J Surg 2004, 91:1111-1124.

19. Buunen M, Veldkamp R, Hop WC, Kuhry E, Jeekel J, Haglind E, Pahlman L, Cuesta MA, Msika S, Morino M, Lacy A, Bonjer HJ: Survival after laparoscopic surgery versus open surgery for colon cancer: long-term outcome of a randomised clinical trial. Lancet Oncol 2009, 10:44-52.

20. Guillou PJ, Quirke P, Thorpe H, Walker J, Jayne DG, Smith AM, Heath RM, Brown JM: Short-term endpoints of conventional versus laparoscopic-assisted surgery in patients with colorectal cancer (MRC CLASICC trial): multicentre, randomised controlled trial. Lancet 2005, 365:1718-1726.

21. Challacombe $B$, Wheatstone $S$ : Telementoring and telerobotics in urological surgery. Curr Urol Rep 2010, 11:22-28.

22. Liao G, Chen J, Ren C, Li R, Du S, Xie G, Deng H, Yang K, Yuan Y: Robotic versus open gastrectomy for gastric cancer: a meta-analysis. PLOS One 2013, 8:e81946.

23. Scandola M, Grespan L, Vicentini M, Fiorini P: Robot-assisted laparoscopic hysterectomy vs traditional laparoscopic hysterectomy: five metaanalyses. J Minim Invasive Gynecol 2011, 18:705-715.

24. Maeso S, Reza M, Mayol JA, Blasco JA, Guerra M, Andradas E, Plana MN: Efficacy of the Da Vinci surgical system in abdominal surgery compared with that of laparoscopy: a systematic review and meta-analysis. Ann Surg 2010, 252:254-262.

25. Yang Y, Wang F, Zhang P, Shi C, Zou Y, Qin H, Ma Y: Robot-assisted versus conventional laparoscopic surgery for colorectal disease, focusing on rectal cancer: a meta-analysis. Ann Surg Oncol 2012, 19:3727-3736.

26. Spinoglio G, Summa M, Priora F, Quarati R, Testa S: Robotic colorectal surgery: first 50 cases experience. Dis Colon Rectum 2008, 51:1627-1632.

27. Deutsch GB, Sathyanarayana SA, Gunabushanam V, Mishra N, Rubach E, Zemon H, Klein JD, Denoto GR: Robotic vs. laparoscopic colorectal surgery: an institutional experience. Surg Endosc 2012, 26:956-963.

28. Baek SJ, Kim SH, Cho JS, Shin JW, Kim J: Robotic versus conventional laparoscopic surgery for rectal cancer: a cost analysis from a single institute in Korea. World J Surg 2012, 36:2722-2729.

29. Patel CB, Ragupathi M, Ramos-Valadez DI, Haas EM: A three-arm (laparoscopic hand-assisted, and robotic) matched-case analysis of intraoperative and postoperative outcomes in minimally invasive colorectal surgery. Dis Colon Rectum 2011, 54:144-150.

30. Poon JT, Law WL: Laparoscopic resection for rectal cancer: a review. Ann Surg Oncol 2009, 16:3038-3047.

31. Kim CW, Kim CH, Baik SH: Outcomes of robotic-assisted colorectal surgery compared with laparoscopic and open surgery: a systematic review. J Gastrointest Surg 2014, 18:816-830.

32. Amato A, Pescatori M: Perioperative blood transfusions for the recurrence of colorectal cancer. Cochrane Database Syst Rev 2006, 7:D5033.

33. Morner ME, Gunnarsson U, Jestin P, Svanfeldt M: The importance of blood loss during colon cancer surgery for long-term survival: an epidemiological study based on a population based register. Ann Surg 2012, 255:1126-1128.

34. Bhangu A, Brown G, Akmal M, Tekkis P: Outcome of abdominosacral resection for locally advanced primary and recurrent rectal cancer. Br J Surg 2012, 99:1453-1461.

35. McPartland KJ, Hyman NH: Damage control: what is its role in colorectal surgery? Dis Colon Rectum 2003, 46:981-986.

36. Mirnezami AH, Mirnezami R, Venkatasubramaniam AK, Chandrakumaran K, Cecil TD, Moran BJ: Robotic colorectal surgery: hype or new hope? A systematic review of robotics in colorectal surgery. Colorectal Dis 2010 12:1084-1093. 
37. Pigazzi A, Luca F, Patriti A, Valvo M, Ceccarelli G, Casciola L, Biffi R, Garcia-Aguilar J, Baek JH: Multicentric study on robotic tumor-specific mesorectal excision for the treatment of rectal cancer. Ann Surg Oncol 2010, 17:1614-1620

38. Keller DS, Senagore AJ, Lawrence JK, Champagne BJ, Delaney CP: Comparative effectiveness of laparoscopic versus robot-assisted colorectal resection. Surg Endosc 2014, 28:212-221.

39. DeSouza AL, Prasad LM, Park JJ, Marecik SJ, Blumetti J, Abcarian H: Robotic assistance in right hemicolectomy: is there a role? Dis Colon Rectum 2010, 53:1000-1006.

40. Halabi WJ, Kang CY, Jafari MD, Nguyen VQ, Carmichael JC, Mills S, Stamos MJ, Pigazzi A: Robotic-assisted colorectal surgery in the United States: a nationwide analysis of trends and outcomes. World I Surg 2013, 37:2782-2790.

41. Aly EH: Robotic colorectal surgery: summary of the current evidence. Int J Colorectal Dis 2014, 29:1-8.

doi:10.1186/1477-7819-12-122

Cite this article as: Liao et al: Robotic-assisted versus laparoscopic colorectal surgery: a meta-analysis of four randomized controlled trials. World Journal of Surgical Oncology 2014 12:122.

\section{Submit your next manuscript to BioMed Central and take full advantage of:}

- Convenient online submission

- Thorough peer review

- No space constraints or color figure charges

- Immediate publication on acceptance

- Inclusion in PubMed, CAS, Scopus and Google Scholar

- Research which is freely available for redistribution 